\title{
DISTRIBUTED COOPERATIVE DATA TRANSFER FOR UWB ADHOC NETWORK
}

\author{
Shouhong Zhu, Kin K. Leung, and Anthony G. Constantinides \\ Communications and Signal Processing Group \\ Department of Electrical and Electronic Engineering \\ Imperial College, London, $\mathrm{UK}^{*}$
}

\begin{abstract}
In this conference paper we address the problem of distributed cooperative data transfer for ultra wide band (UWB) ad-hoc networks: 1) we propose three improving techniques that can utilize some available but not-yet-utilized additional resources to improve the sub-optimal but widely adopted autocorrelation differential detection for the widely used pulse-based UWB data transfer; 2) we devise three cooperative transmission strategies, and discuss the necessary information needed for distributed implementations and how it may be obtained via proposed transmission protocols. Simulation studies confirm the effectiveness of the proposed techniques and schemes.
\end{abstract}

\section{INTRODUCTION}

The recently regulated unlicensed use of the ultra wide band (UWB) radio spectrum provides wireless ad-hoc networks a low-cost radio technology for their large-scale applications [1][5]. Furthermore, the use of cooperative transmission strategies lead to significantly improved performance on the data transfer carried out within a wireless ad-hoc network [6]. However, to practtically realize efficient cooperative data transfer we need the cross-layer optimized protocols that can be implemented in a distributed way [7]-[9].

In this paper we first propose three improving techniques that can utilize some available but not-yet-utilized additional resources to improve the sub-optimal but widely adopted autocorrelation differential detection for the widely used pulsebased UWB data transfer, and then devise three cooperative transmission strategies: data relaying via routing, data relaying via transmit-beamforming, and multiple source / input multiple destination / output (MIMO) data relaying via orthogonalization, and also discuss the necessary information that is needed for distributed implementations and how it may be obtained via proposed transmission protocols.

\section{Improve PUlse-BASEd UWB DATA TRANSFER}

In UWB ad-hoc networks the UWB data communications widely use the pulse-based UWB data transfer, for which the autocorrelation differential detection is a sub-optimal but widely adopted data detection approach, thanks to its simplicity due to avoiding channel estimation and thus resultant outstanding immunity to fast channel variation. In this section we first present the signal model for the pulse-based UWB data transfer, and then propose three improving techniques to improve such autocorrelation differential detection.

\footnotetext{
${ }^{*}$ This work was supported by the EU-funded PULSERS research project.
}

Consider a source UWB node sends data via applying the binary pulse amplitude modulation (BPAM) on a train of ultrashort UWB pulses, its destination UWB node would receive the signal that can be represented as follows

$$
\begin{aligned}
r(t) & =\sum_{k} b_{k} \sum_{n_{f}=0}^{N_{f}-1} p\left(t-k T_{s}-n_{f} T_{f}\right) * h(t-\tau)+w(t) \\
& =\sum_{k} b_{k} \sum_{n_{f}=0}^{N_{f}-1} p_{h}\left(t-\tau-k T_{s}-n_{f} T_{f}\right)+w(t)
\end{aligned}
$$

where $b_{k}$ is the transmitted binary data symbol at time index $k ; p(t)$ is the ultra-short UWB pulse which is normalized and has effective width $T_{p} ; T_{s}=N_{f} T_{f}$ is the data symbol duration in which there are $N_{f}$ frames, each with frame duration $T_{f}$; to avoid any inter symbol interference (ISI) the frame duration is designed to be wider than the maximum channel delay spread $T_{h}$; the character $*$ refers to the convolution operator; $h(t)$ is the channel impulse response; $\tau$ is the signal transmission delay with respect to the receiver's clock, and is assumed without loss of generality that $0 \leq \tau<T_{s} ; w(t)$ is the received noise which is assumed to be white zero-mean and circularly symmetric complex Gaussion noise with power spectral density $N_{o}$. As the binary data symbols are independent and identically distributed (iid) and are drawn from $\{ \pm 1\}$, their received bit energy to noise power spectral density ratio would be

$$
E_{b} / N_{o}=\frac{N_{f} \int_{0}^{T_{f}}\left|p_{h}(t)\right|^{2} d t}{N_{o}}=\gamma
$$

The ideal data detection approach is the channel-matched data detection, in which the channel is synchronized and estimated first and then correlates with the received signal

$$
\begin{aligned}
x(k, \tau)= & \int_{\tau+k T_{s}}^{\tau+(k+1) T_{s}} \sum_{n_{f}=0}^{N_{f}-1} p_{h}^{*}\left(t-\tau-k T_{s}-n_{f} T_{f}\right) r(t) d t \\
= & b_{k} N_{f} \int_{0}^{T_{f}}\left|p_{h}(t)\right|^{2} d t+ \\
& +\int_{0}^{T_{s}} \sum_{n_{f}=0}^{N_{f}-1} p_{h}^{*}(t) w\left(t+\tau+k T_{s}+n_{f} T_{f}\right) d t
\end{aligned}
$$

therefore $b_{k}$ could be detected based on the real part of the above calculation, i.e.

$$
\widehat{b}_{k}=\operatorname{sign}\{\operatorname{Re}[x(k, \tau)]\}
$$

The data symbols in such obtained statistic for detection have their SNR as

$$
\operatorname{SNR}_{\text {detection }}=\frac{N_{f} \int_{0}^{T_{f}}\left|p_{h}(t)\right|^{2} d t}{N_{o} / 2}=2 \gamma
$$


For fast varying/fading UWB channels, the channel-matched detection is computationally complicated, whereas the autocorrelation differential detection is a sub-optimal but widely adopted data detection approach. It does not need to estimate the channel except for the data symbol timing. In such autocorrelation differential detection the received signal is the autocorrelations at one data symbol lag, i.e.

$$
y(k, \widetilde{\tau})=\int_{\widetilde{\tau}+k T_{s}}^{\tilde{\tau}+(k+1) T_{s}} r^{*}\left(t-T_{s}\right) r(t) d t
$$

If synchronization is achieved, i.e. $\widetilde{\tau}=\tau$, the above would be

$$
\begin{aligned}
y(k, \tau)= & b_{k-1} b_{k} N_{f} \int_{0}^{T_{f}}\left|p_{h}(t)\right|^{2} d t \\
& +b_{k-1} \int_{0}^{T_{s}} \sum_{n_{f}=0}^{N_{f}-1} p_{h}^{*}(t) w\left(t+\tau+k T_{s}+n_{f} T_{f}\right) d t \\
+ & b_{k} \int_{0}^{T_{s}} \sum_{n_{f}=0}^{N_{f}-1} p_{h}(t) w^{*}\left(t+\tau+(k-1) T_{s}+n_{f} T_{f}\right) d t \\
& +\int_{0}^{T_{s}} w^{*}\left(t+\tau+(k-1) T_{s}\right) w\left(t+\tau+k T_{s}\right) d t
\end{aligned}
$$

the differential data symbol $b_{k-1} b_{k}=q_{k-1, k}$ could be detected based on the real part of the above calculation, i.e.

$$
\widehat{q}_{k-1, k}=\operatorname{sign}\{\operatorname{Re}[y(k, \tau)]\}
$$

The multiplication of two consecutive data symbols in such obtained statistic for detection have their SNR as

$$
\mathrm{SNR}_{\text {detection }}=\frac{N_{f} \int_{0}^{T_{f}}\left|p_{h}(t)\right|^{2} d t}{N_{o}+\frac{N_{o}^{2} N_{f} T_{f}}{2 T_{p}}}=\frac{\gamma}{1+\frac{N_{f} T_{f}}{2 \gamma T_{p}}}
$$

where we can see that the real part of the non-Gaussion 'double-noise' item has adverse effects in data detection. And this is why the autocorrelation differential detection suffers from performance deterioration as comparing with the channelmatched differential detection.

Below we propose three improving techniques to improve the promising autocorrelation differential detection.

\section{A. Narrowing Integration Period}

Normally, the integration period in each frame is the whole frame duration. Considering that the maximum channel delay spread is a priori known shorter than the frame duration, so narrowing the integration period in each frame to the maximum channel delay spread would improve the SNR of the data symbols in the obtained statistic for detection

$$
\mathrm{SNR}_{\text {detection }}=\frac{\gamma}{1+\frac{N_{f} T_{h}}{2 \gamma T_{p}}}
$$

Comparing with equation (9), the above receiver SNR has less adverse effects caused by the 'double-noise' items.

\section{B. Utilizing Additional Cross-Frame Autocorrelations}

Within data symbol duration there are $N_{f}$ pieces of received signal corresponding to $N_{f}$ frames respectively. So we can have $N_{f}^{2}$ independent inter-frame correlations rather than $N_{f}$ ones. The independence here refers to that of the non-Gaussian 'double-noise' items involved. If taking advantages of all interframe correlations within data symbol duration, we can obtain a statistic for detection that has improved receiver SNR as

$$
\mathrm{SNR}_{\text {detection }}=\frac{\gamma}{1+\frac{T_{f}}{2 \gamma T_{p}}}
$$

Comparing with equation (9), the above receiver SNR has less adverse effects caused by the 'double-noise' items.

\section{Utilizing Additional Autocorrelations at Larger Lag}

Look at the autocorrelation differential detection on $q_{k-1, k}=$ $b_{k-1} b_{k}$ and $q_{k, k+1}=b_{k} b_{k+1}$, we can see that $q_{k-1, k+1}=$ $q_{k-1, k} q_{k, k+1}$ also could be detected based on the measurably available autocorrelations at the lag of two data symbol durations. So definitely the previously detected $\widehat{q}_{k-1, k}$ and $\widehat{q}_{k, k+1}$ could be error-corrected by the independently detected $\widehat{q}_{k-1, k+1}$. In the same way, the additional autocorrelations at the lag of more data symbols would further improve the data detection performance.

Obviously, ideal error-correcting algorithm with these additional autocorrelations at larger lag would improve data detection performance greatly. To efficiently utilize the additional autocorrelations at the lag of two data symbols, we propose a simple approach which does not increase too much the computational complexity: the detected $\widehat{q}_{k-1, k}$ from the autocorrelation at one data symbol duration lag would change its sign only in the case it causes the following two inequities simultaneously

$$
\begin{aligned}
& \widehat{q}_{k-2, k-1} \widehat{q}_{k-1, k} \neq \widehat{q}_{k-2, k} \\
& \widehat{q}_{k-1, k} \widehat{q}_{k, k+1} \neq \widehat{q}_{k-1, k+1}
\end{aligned}
$$

\section{CoOperative DATA TRANSFER ANd FEAsible DISTRIBUTED IMPLEMENTATIONS}

Cooperative data transfer refers to such an enhanced data transfer as to add relaying-based collaborative transmissions on the normal direct transmission. In this section we discuss three cooperative transmission strategies: data relaying via routing, data relaying via transmit-beamforming, and MIMO data relaying via orthogonalization.

\section{A. Data Relaying via Routing}

Data relaying via routing can be shown as in Fig.1. A source node broadcasts data in the first time slot to its destination node as well as all available relaying nodes, and then in the second time slot the data is forwarded to the destination node by the best relay. The 'best relay' here means the relay associating with the highest instantaneous route quality.

The basis for cooperative data transfer via routing is the following obvious remark 


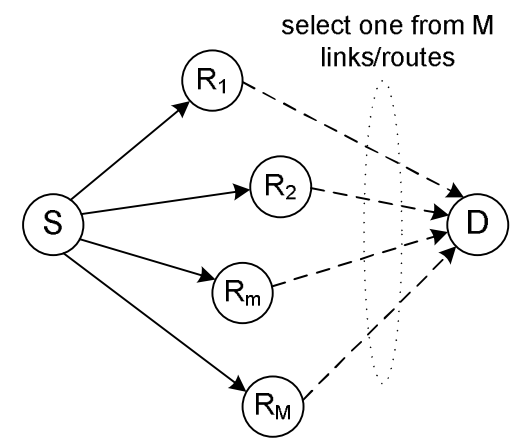

Figure 1: Data relaying via routing.

Remark 1 If the asymptotic outage probability (AOP) of each $U W B$ relaying route is $\chi \frac{1}{\bar{\rho}^{\gamma}}$, then the AOP of the qualityselected $U W B$ relaying route from $M$ available ones is

$P_{\text {outage }}^{(\text {as })}=\operatorname{Pr}^{(\bar{\rho} \rightarrow \infty)}\{\rho<1\}=\operatorname{Pr}^{(\bar{\rho} \rightarrow \infty)}\left\{\frac{\rho}{\bar{\rho}}<\frac{1}{\bar{\rho}}\right\}=\left(\chi \frac{1}{\bar{\rho}^{\gamma}}\right)$

This remark is obvious because

$$
\begin{aligned}
\operatorname{Pr}\{\rho<1\} & =\operatorname{Pr}\left\{\rho_{m}<1, \quad m=1, \cdots, M\right\} \\
& =\operatorname{Pr}\left\{\rho_{1}<1\right\} \cdots \operatorname{Pr}\left\{\rho_{M}<1\right\} \\
& =\operatorname{Pr}\left\{\frac{\rho_{1}}{\bar{\rho}}<\frac{1}{\bar{\rho}}\right\} \cdots \operatorname{Pr}\left\{\frac{\rho_{M}}{\bar{\rho}}<\frac{1}{\bar{\rho}}\right\}
\end{aligned}
$$

where the independence among relaying routes is utilized.

From this remark we can see that with the additional qualityselected routing the cooperative data transfer exhibits an increased diversity order as compared to the direct data transfer or the random single relaying based data transfer.

The protocol that is to realize distributed cooperative routing scheme could work as follows: all relaying nodes first receive the signal transmitted from the source node and detect the data independently; immediately after receiving the last data symbol from the source node, each relaying node starts monitoring the medium via an enhanced UWB pulse-sensing (to deal with the problem of hidden nodes, the UWB pulse-sensing is enhanced by adding a quick acknowledge signal bounced from the destination node once it receives the first data symbol from any relaying node); if the medium is occupied by any other relaying node for data forwarding or by the quick acknowledge signal bounced from the destination node, the relaying node aborts its prepared but not-yet-started data forwarding; each relaying node determines its associated source-relay-destination route quality in a distributed way by estimating the receiver SNR of its source-relay link and/or by acquiring the receiver SNR of its relay-destination link; each relaying node deterministically maps its associated source-relay-destination route quality onto its back-off period, and sets a down-counted timer with it, and when the time goes up, starts its data forwarding.

Another important issue in distributed implementations is how to optimally map the relaying route quality to its corresponding back-off period.

We know if the duration needed for relays to sense and detection the occupation of media is $\Delta$ and the mapped back-off periods of each relay exhibits a distribution whose probability density function (pdf) is $f(\cdot)$, then for $M$ relays the collision probability would be

$$
p_{c}=1-\int_{0}^{+\infty} c(x) d x
$$

where $c(\cdot)$ is the pdf of the distribution of the shortest period that is shorter than others by $\Delta$ (thus no collision occurs)

$$
c(x)=M f(x)[1-F(x+\Delta)]^{M-1}
$$

where $F(\cdot)$ is the cumulative function of $f(\cdot)$. Considering

$$
\int_{0}^{+\infty} M f(x)[1-F(x)]^{M-1} d x=1
$$

we can see that the smallest collision probability with given $\Delta$ could be achieved only in the case the mapped back-off periods exhibit uniform distribution. If the mapped back-off periods exhibit such uniform distribution, say

$$
f(x)=\frac{1}{T}, x \in[0, T]
$$

where $T$ is the range of $x$, the collision probability would be

$$
p_{c} \approx M \Delta^{M-1} \int_{0}^{+\infty} f^{M}(x) d x=M\left(\frac{\Delta}{T}\right)^{M-1}
$$

Meanwhile, on the other hand, the average data relaying delay can be calculated as follows

$$
T_{a d}=\int_{0}^{+\infty} x g(x) d x
$$

where $g(\cdot)$ is the pdf of the distribution of the shortest period

$$
g(x)=M f(x)[1-F(x)]^{M-1}
$$

If the mapped back-off periods exhibit uniform distribution, then

$$
T_{a d}=\frac{T}{M+1}
$$

From the relationship between the collision probability and the average data relaying delay we can see that decreasing the acceptable average data relaying delay would simply result in increasing the achieved collision probability, or vice versa. So we can plan the achievable collision probability according to our acceptable average data relaying delay.

\section{B. Data Relaying via Transmit-Beamforming}

Data relaying via transmit-beamforming can be shown as in Fig.2. A source node broadcasts data in the first time slot to its destination node as well as all available relaying nodes, and then in the second time slot the data is forwarded to the destination node by combining the contributions from all available relays.

When the source node broadcasts its data to relays, the received signal at relay $m$, after pre-processing, can be represented as

$$
r_{m}(k)=h_{s m} \sqrt{P_{s}} b_{k}+n_{m}(k)
$$




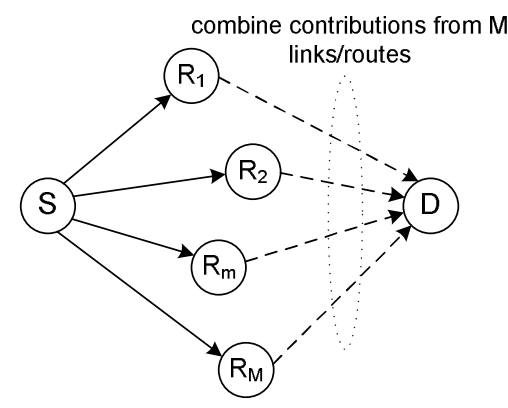

Figure 2: Data relaying via beamforming.

Under the decode-and-forward data relaying policy, the relayed signal at relay $m$ is

$$
s_{m}(k)=\widehat{b}_{k}=\operatorname{sign}\left\{\operatorname{Re}\left[r_{m}(k) e^{-j \widehat{\phi}_{s m}}\right]\right\}
$$

where $\widehat{\phi}_{s m}$ is the estimated phase of the link gain $h_{s m}$. If the transmit weight for such relaying is assigned as $w_{m}$ with the total limited transmit power $\sum_{m}\left|w_{m}\right|^{2}=P_{s}$, then we have the received signal at the destination node as follows

$$
r_{d l}(k)=\sum_{m} h_{m d l} w_{m} s_{m}(k)+n_{d l}(k)
$$

where $l$ is the delay-separable path index. Considering that there maybe detection errors at relays, if we assume the error probability for the data detection at relay $m$ is

$$
e_{m}=\mathrm{Q}\left(\frac{2 P_{s}\left|h_{s m}\right|^{2}}{\sigma_{n}^{2}}\right)
$$

where $Q($.$) is the error function, then we have$

$$
s_{m}(k)=\widehat{b}_{k}=\left\{\begin{aligned}
b_{k} & \text { with } \operatorname{Pr}=1-e_{m} \\
-b_{k} & \text { with } \operatorname{Pr}=e_{m}
\end{aligned}\right\}
$$

The resulting SNR at the destination node is therefore

$$
\mathrm{SNR}=\frac{\sum_{l}\left[\sum_{m} h_{m d l} w_{m}\left(1-2 e_{m}\right)\right]^{2}}{\sigma_{n}^{2} / 2}
$$

Here due to the limited space we only consider the case with single-path channels and synchronized combination. So when we optimize the transmit weights as

$$
w_{m}=\frac{h_{m d}^{*}\left(1-2 e_{m}\right) \sqrt{P_{s}}}{\sqrt{\sum_{m}\left|h_{m d}\right|^{2}\left(1-2 e_{m}\right)^{2}}}, m
$$

then the corresponding maximum achievable SNR at the destination node can be obtained accordingly

$$
\mathrm{SNR}=\frac{2 P_{s}}{\sigma_{n}^{2}} \sum_{m}\left|h_{m d}\right|^{2}\left(1-2 e_{m}\right)^{2}
$$

We can see that with the additional transmit-beamformingbased relaying the cooperative data transfer exhibits an increased diversity order as compared to the direct data transfer or the random single relaying based data transfer.

\section{MIMO Data Relaying via Orthogonalization}

MIMO data relaying via orthogonalization can be shown as in Fig.3. I source nodes broadcast data in the first time slot to their $J$ destination nodes as well as all available relaying nodes, and then the data are forwarded to their destination nodes by combining the contributions from all available relays. Here due to the limited space all channels are assumed to be single-path channels.

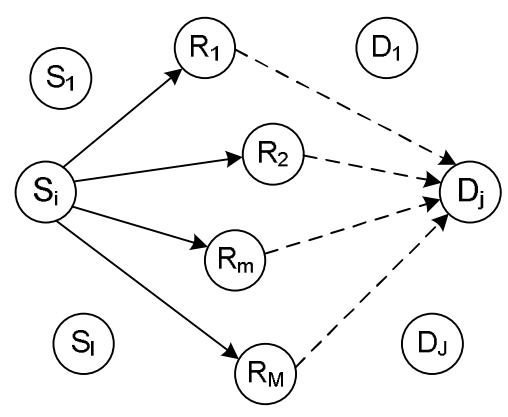

Figure 3: MIMO data relaying via orthogonalization.

The received signals at relays in vector form, $\mathbf{r}_{r}(k)=$ $\left[r_{r 1}(k) \cdots r_{r M}(k)\right]^{T}$, after pre-processing, can be represented as

$$
\mathbf{r}_{r}(k)=\mathbf{H}_{s r} \sqrt{P_{s}} \mathbf{s}_{s}(k)+\mathbf{n}_{r}(k)
$$

where $\mathbf{s}_{s}(k)=\left[b_{1}(k) \cdots b_{I}(k)\right]^{T}$ is the vector composed of data symbols transmitted from source nodes, $P_{s}$ is the transmit power of each source node, $\mathbf{H}_{s r}$ is the matrix composed of complex channel gains from source nodes to relays, and $\mathbf{n}_{r}(k)=\left[n_{r 1}(k) \cdots n_{r M}(k)\right]^{T}$ is the vector composed of received noises at relays.

Under the amplify-and-forward data relaying policy, the retransmitted signals from relays, $\mathbf{s}_{r}(k)=\left[s_{r 1}(k) \cdots s_{r M}(k)\right]^{T}$ in vector form, are

$$
\mathbf{s}_{r}(k)=\mathbf{W} \mathbf{r}_{r}(k)
$$

where $\mathbf{W}$ is a diagonal matrix whose parameters satisfy

$$
\begin{aligned}
\mathrm{E}\left\{\left\|\mathbf{s}_{r}(k)\right\|^{2}\right\} & =\operatorname{tr}\left\{\mathbf{W} \mathbf{H}_{s r} \mathbf{H}_{s r}^{H} \mathbf{W}^{H}\right\} P_{s}+\operatorname{tr}\left\{\mathbf{W} \mathbf{W}^{H}\right\} \sigma_{n}^{2} \\
& =I P_{s}
\end{aligned}
$$

i.e. the requirement on total transmit power.

The received signals at destination nodes are therefore

$$
\begin{aligned}
\mathbf{r}_{d}(k) & =\mathbf{H}_{r d} \mathbf{W} \mathbf{s}_{r}(k)+\mathbf{n}_{d}(k) \\
& =\mathbf{H}_{r d} \mathbf{W} \mathbf{H}_{s r} \sqrt{P_{s}} \mathbf{s}_{s}(k)+\mathbf{H}_{r d} \mathbf{W} \mathbf{n}_{r}(k)+\mathbf{n}_{d}(k)(35)
\end{aligned}
$$

where $\mathbf{r}_{d}(k)=\left[r_{d 1}(k) \cdots r_{d J}(k)\right]^{T}$, and $\mathbf{H}_{r d}$ is the matrix composed of channel gains from relays to destination nodes, and $\mathbf{n}_{d}(k)=\left[n_{d 1}(k) \cdots n_{d J}(k)\right]^{T}$ is the vector composed of received noises at destination nodes.

The problem is that: can we optimize the diagonal matrix $\mathbf{W}$, actually its diagonal parameters, such that

$$
\mathbf{H}_{r d} \mathbf{W H}_{s r}=\mathbf{V}
$$


where $\mathbf{V}$ is a diagonal matrix, i.e. MIMO relaying with zeroforcing orthogonalization. The answer to this question is definitely 'yes', if the number of relays is sufficient.

Due to the limited space, we just give following examples: 1. in the case with totally two end-nodes forming one two-way data transfer link, one relay is needed for orthogonalization; 2. in the case with totally four end-nodes forming two one-way data transfer links, four relays are needed for orthogonalization; 3 . in the case with totally four end-nodes forming one two-way and one one-way data transfer links, six relays are needed for orthogonalization.

\section{Simulation Studies}

First, we evaluate the performance of the improved (noncoherent) autocorrelation differential detection. The simulation results, obtained by averaging over 100,000 independent runs, are shown in Fig.4. In the simulations it is assumed the maximum channel delay spread is 6 pulse durations, the data symbol duration contains 16 frames, each frame contains 16 pulse durations.

From the figure we can see that the proposed improving techniques can be used, separately or jointly, to significantly improve the performance of the autocorrelation differential detection.

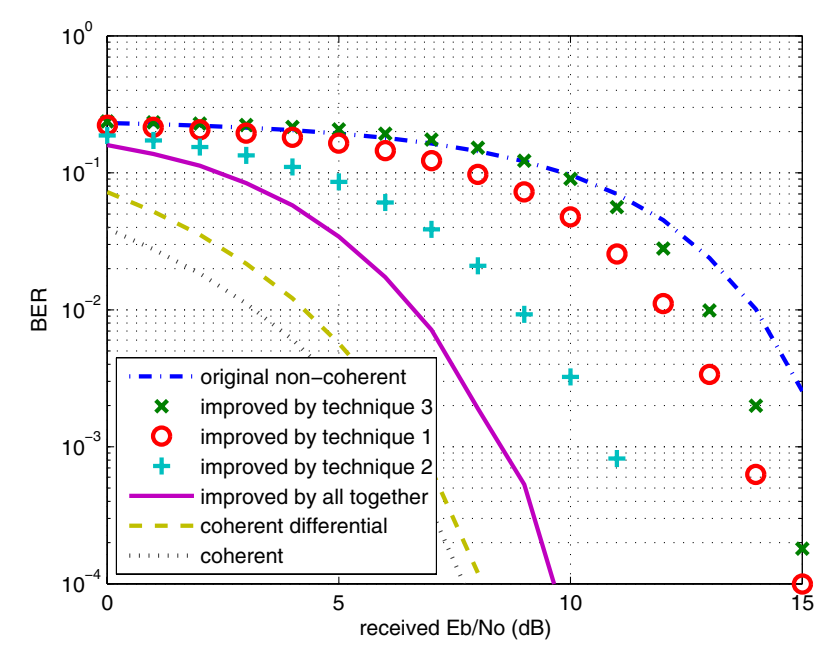

Figure 4: Performance of the improved (non-coherent) autocorrelation differential detection.

Second, we evaluate the performance of the proposed distributed cooperative data transfer schemes. The simulation results, obtained by averaging over 1000,000 independent runs, are shown in Fig.5. Here the phrase 'no feedback' means the selection is only based on source-relay link quality; the phrase '1-bit feedback (5 10 15)' means the relay-destination link SNR is assigned as 5 if less than 10 and 15 otherwise; the phrase '2-bit feedback (phase)' means the relay-destination link phase is assigned with error in $\pm 45^{\circ}$.

From the figure we can see that the proposed distributed cooperative transmission schemes can significantly improve the data transfer performance.

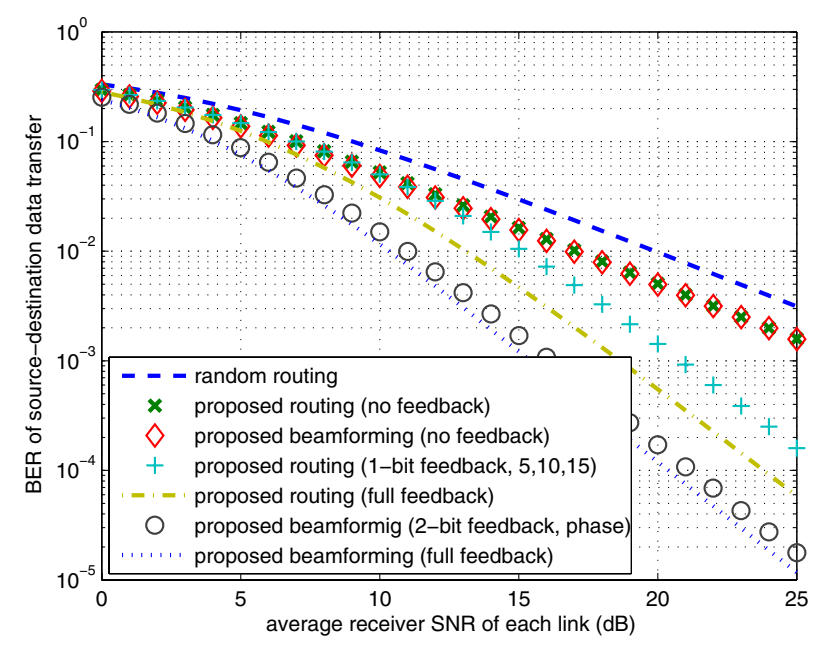

Figure 5: Performance of the proposed distributed cooperative data transfer.

\section{CONCLUSION}

We have studied following issues: 1) three improving techniques to improve the promising autocorrelation differential detection for the widely used pulse-based UWB data transfer; 2) three cooperative transmission strategies and the necessary information needed for distributed implementations. Simulation studies have confirmed the effectiveness of the proposed techniques and schemes.

\section{REFERENCES}

[1] C. Siva Ram Murthy and B. S. Manoj, Ad-Hoc Wireless Networks: Architectures and Protocols, Prentice Hall, 2004

[2] http://www.uwbforum.org - The website for the UWB Forum

[3] D. Porcino and W. Hirt, "Ultra wideband radio technology: Potential and challenges ahead", IEEE Communications Magazine, vol.4, no.7, Jul. 2003

[4] http://www.pulsers.eu - The website for the EU funded PULSERS research project

[5] http:// www.commsp.ee.ic.ac.uk/ wiser/pulsers/ — The website for the EU funded PULSERS research project at Imperial College

[6] J. N. Laneman, D. N. C. Tse, and G. W. Wornell, "Cooperative diversity in wireless networks: Efficient protocols and ourage behavior", IEEE Transaction on Information Theory, vol.51, no.12, Dec. 2004

[7] Aggelos Bletsas, Ashish Khisti, David P. Reed, and Andrew Lippman, "A simple cooperative diversity method based on network path selection", IEEE Journal on selected areas of communications, Vol.24, No.3, 2006

[8] Cemal Akcaba, Rohit U. Nabar, and Kin K. Leung, "A PHY/MAC approach to wireless routing", Proc. of IEEE ICC2006, 2006

[9] Shouhong Zhu and Kin K. Leung, "Distributed cooperative routing for UWB ad-hoc networks", Proc. of IEEE ICC2007, Glasgow, UK, 2007 